\title{
BMJ Open Protocol for a multicentre study to assess feasibility, acceptability, effectiveness and direct costs of TRIumPH (Treatment and Recovery In PsycHosis): integrated care pathway for psychosis
}

To cite: Rathod S, Garner C, Griffiths $A$, et al. Protocol for a multicentre study to assess feasibility, acceptability, effectiveness and direct costs of TRlumPH (Treatment and Recovery In PsycHosis): integrated care pathway for psychosis. BMJ Open 2016:6:e012751.

doi:10.1136/bmjopen-2016012751

- Prepublication history for this paper is available online. To view these files please visit the journal online (http://dx.doi.org/10.1136/ bmjopen-2016-012751).

Received 23 May 2016 Revised 4 July 2016 Accepted 2 August 2016

CrossMark

For numbered affiliations see end of article.

Correspondence to Dr Shanaya Rathod; shanayarathod@nhs.net

\section{ABSTRACT}

Introduction: Duration of untreated psychosis (time between the onset of symptoms and start of treatment) is considered the strongest predictor of symptom severity and outcome. Integrated care pathways that prescribe timeframes around access and interventions can potentially improve quality of care.

Methods and analysis: A multicentre mixed methods study to assess feasibility, acceptability, effectiveness and analysis of direct costs of an integrated care pathway for psychosis. A pragmatic, non-randomised, controlled trial design is used to compare the impact of Treatment and Recovery In PsycHosis (TRlumPH; Intervention) by comparison between NHS organisations that adopt TRlumPH and those that continue with care as usual (Control). Quantitative and qualitative methods will be used. We will use routinely collected quantitative data and studyspecific questionnaires and focus groups to compare service user outcomes, satisfaction and adherence to intervention between sites that adopt TRlumPH versus sites that continue with usual care pathways.

Setting: 4 UK Mental health organisations. Two will implement TRlumPH whereas two will continue care as usual.

Participants: Staff, carers, individuals accepted to early intervention in psychosis teams in participating organisations for the study period.

Intervention: TRlumPH — Integrated Care Pathway for psychosis that has a holistic approach and prescribes time frames against interventions; developed using intelligence from data; co-produced with patients, carers, clinicians and other stakeholders.

Outcomes: Feasibility will be assessed through adherence to the process measures. Satisfaction and acceptability will be assessed using questionnaires and focus groups. Effectiveness will be assessed through data collection and evaluation of patient outcomes,

\section{Strengths and limitations of this study}

- Robust methodology used for development of the pathway;

- One of the few service evaluation studies;

- Evaluation of direct costs is an integral part of the study;

- Results will be generalisable to NHS organisations, but there may be challenges in international adoption;

- Limited resources within services to deliver care along the pathway may impact on results.

including clinical, functional and recovery outcomes, physical health, acute care use. Outcome measures will be assessed at baseline, 12 and 24 months to measure whether there is an effect and if so, whether this is sustained over time. Outcomes measures at the adopter sites will be compared to their own baseline and against comparator sites.

Ethics and dissemination: Ethics approval was obtained from East of Scotland Research Ethics Service (REC Ref no: LR/15/ES/0091). The results will be disseminated through publications, conference presentations, reports to the organisation.

Study registration: UK Clinical Research Network Portfolio: 19187.

\section{BACKGROUND}

Pooled incidence of all psychoses in the UK is documented at 31.7/100 000 person-years. ${ }^{1}$ Evidence suggests that first episode psychosis occurs more often in young men; a secondary peak in the first episode is seen in 
women around the age of 45 years. The occurrence of new episodes is higher in some black and minority ethnic groups. Psychosis is more common in urban populations, particularly in deprived areas. The course is usually recurrent, with significant health service use (and associated costs), especially at 'crisis' points with inpatient bed usage. ${ }^{12}$ Life expectancy is reduced by $\sim 15-20$ years, mostly as a consequence of physical health problems. ${ }^{3}$

Schizophrenia, the most burdensome of psychoses, is among the most costly illnesses worldwide. According to the Global Burden of Disease Study, schizophrenia causes a high degree of disability, which accounts for $1.1 \%$ of the total disability-adjusted life years (DALYs) and $2.8 \%$ of years lived with disability (YLDs). In the WHO report, schizophrenia is listed as the 8th leading cause of DALYs worldwide in the age group 1544 years. ${ }^{4}$ In addition to the direct cost, there is a considerable burden on the relatives. ${ }^{5}$

The longer the time between the onset of symptoms of psychosis and the start of treatment-known as the duration of untreated psychosis (DUP) - the worse the outlook, more so for social functioning and recovery. ${ }^{6} 7$ Therefore, DUP is considered to be the strongest predictor of symptom severity and prognosis. ${ }^{8}$ Evidence from trans-cultural and international research suggests that DUP ranges between 364 and 721 days $^{9} 10$ and reducing DUP is of imminent national and international importance. ${ }^{4}$ Therefore, the pathway goals are to identify the illness as early as possible, treat the symptoms, provide skills to patients and their families, maintain the improvement over a period, prevent relapses and reintegrate the individuals into the community so that they can lead as normal a life as possible. ${ }^{5}$

Longer durations of untreated psychosis can occur due to client-related or service-related factors, or both. Client-related factors could be attributable to an inability to recognise early symptoms or due to the illness itself such as poor insight, social and emotional withdrawal ${ }^{11}$ or due to cultural values systems like attributions to illness and help-seeking pathways into care. ${ }^{12} 13$

There is a considerable unmet need currently in patients with psychosis in the way they can access services and receive evidence-based treatments as prescribed by the National Institute for Health and Care Excellence $(\mathrm{NICE})^{14}$ in a timely fashion. The Schizophrenia Commission Report ${ }^{15}$ and National Audit of Schizophrenia ${ }^{16}$ have highlighted deficiencies in psychopharmacological and psychological care and recommended changes to the way care is provided to patients with schizophrenia. There is strong cost-effectiveness argument supporting early intervention and early intervention in psychosis (EIP) teams were developed in many countries to achieve this goal, but, in attempts to reduce costs, these teams have been diluted in many areas. ${ }^{17}$ Community education and awareness campaigns have been tried to identify symptoms early with limited success. ${ }^{18}$ Educating general practitioners to reduce delays in referrals to secondary care services has had limited impact ${ }^{19}$ in increasing the number of referrals. Delays in secondary care mental health services have been found to be of most significance in the care pathways of those with long DUP. ${ }^{20} 21$

Therefore, there is a need for integrated pathways prescribing key stages, timelines and interventions matched to the diversity of presentations that occur with psychosis, for example, comorbidity with substance misuse and trauma, which will improve access to evidence-based services, interventions and support recovery.

Integrated pathways are also fundamental to improving the physical health and reducing premature morbidity and mortality in people with psychosis. Pathways can ensure delivery of appropriate health promotion at key stages, for example, weight gain from antipsychotic medication is especially pronounced in the first 8 weeks of administration but it is rare that specific support is offered at this stage. Similarly smoking cessation support can be built in at critical periods, for example, on admission to hospital. Integrated pathways improve the quality of care by improving multidisciplinary communication, care planning, including primary care, and patient satisfaction. $^{22}$

\section{RESEARCH QUESTION}

Is implementation of TRIumPH integrated pathway in services feasible and acceptable and does it reduce duration of untreated psychosis, hospital stay and severity of symptoms at 12 and 24 months?

\section{Study objectives}

The primary objective of this study was to assess the feasibility, acceptability and effectiveness of the psychosis pathway, TRIumPH. The secondary objective is to conduct an evaluation of direct costs and to explore barriers to implementation of the pathway with staff.

\section{METHODS}

\section{Study design}

This is a multicentre, non-randomised, mixed-method study to evaluate feasibility, acceptability, effectiveness and analysis of direct costs (eg, care cost, hospital bed costs etc) of an integrated care pathway, TRIumPH. Qualitative and quantitative data will be collected and analysed.

\section{Setting}

The study has two adopter organisations-early intervention in psychosis teams in Southern Health NHS Foundation Trust and Sheffield Health and Social Care NHS Foundation Trust and two comparator organisations-EIP teams in Solent NHS Trust and Dorset Healthcare University NHS Foundation Trust. 


\section{The intervention: TRlumPH-integrated care pathway}

TRIumPH is an integrated care pathway for psychosis that prescribes time frames around access and clinical interventions as developed in the UK (figures 1 and 2).$^{23}$ The work has used a similar approach to that taken to improve care in other health areas like stroke care, where there has been a demonstrable improvement in outcomes for patients and carers. This new psychosis pathway aims to reduce the impact of disease and promote recovery by ensuring that every individual gets the best evidence-based care at the right time and in the right place.

In developing the pathway, a multipronged approach has been used, using (i) intelligence from information, (ii) co-production with individuals with lived experience of mental illness and their carers and (iii) engagement with clinicians and other stakeholders, including commissioners, primary care and third-sector organisations. The approach has used a robust methodology that can be adapted and adopted nationally and internationally.

\section{Intelligence from information}

In developing the psychosis pathway, the Wessex Academic Health Sciences Network (AHSN) commissioned Janssen Healthcare Innovation to build a comprehensive picture of service use in Wessex from national data sets by pseudonymising, non-sensitive patient-level data for 2012-2013. Licensed from the Health and Social Care Information Centre, the Mental Health Minimum Data Set (MHMDS), and Hospital Episode Statistics (HES) were searched for individuals identified using the relevant ICD-10 clinical codes; Mental health (Payment by Results) PbR clusters and Health of the Nation Outcome Scale (HoNOS). The data confirmed that individuals who suffered with psychosis: $^{23}$

- Had higher psychiatric hospital admissions with longer stays (on average over $50 \%$ longer than other mental health conditions);

- Accounted for the majority of community health professional contacts (around three times more);

- Had more attendances and admissions to local accident and emergency departments

- Are keen to work but could not find employment.

Limitations with the data and method used were acknowledged. The information on service use was consistent with other areas in the country. ${ }^{23}$

\section{Coproduction}

The Wessex AHSN supported co-production workshops that enabled patient, carer and clinician feedback. The aim of the coproduction workshops was to understand

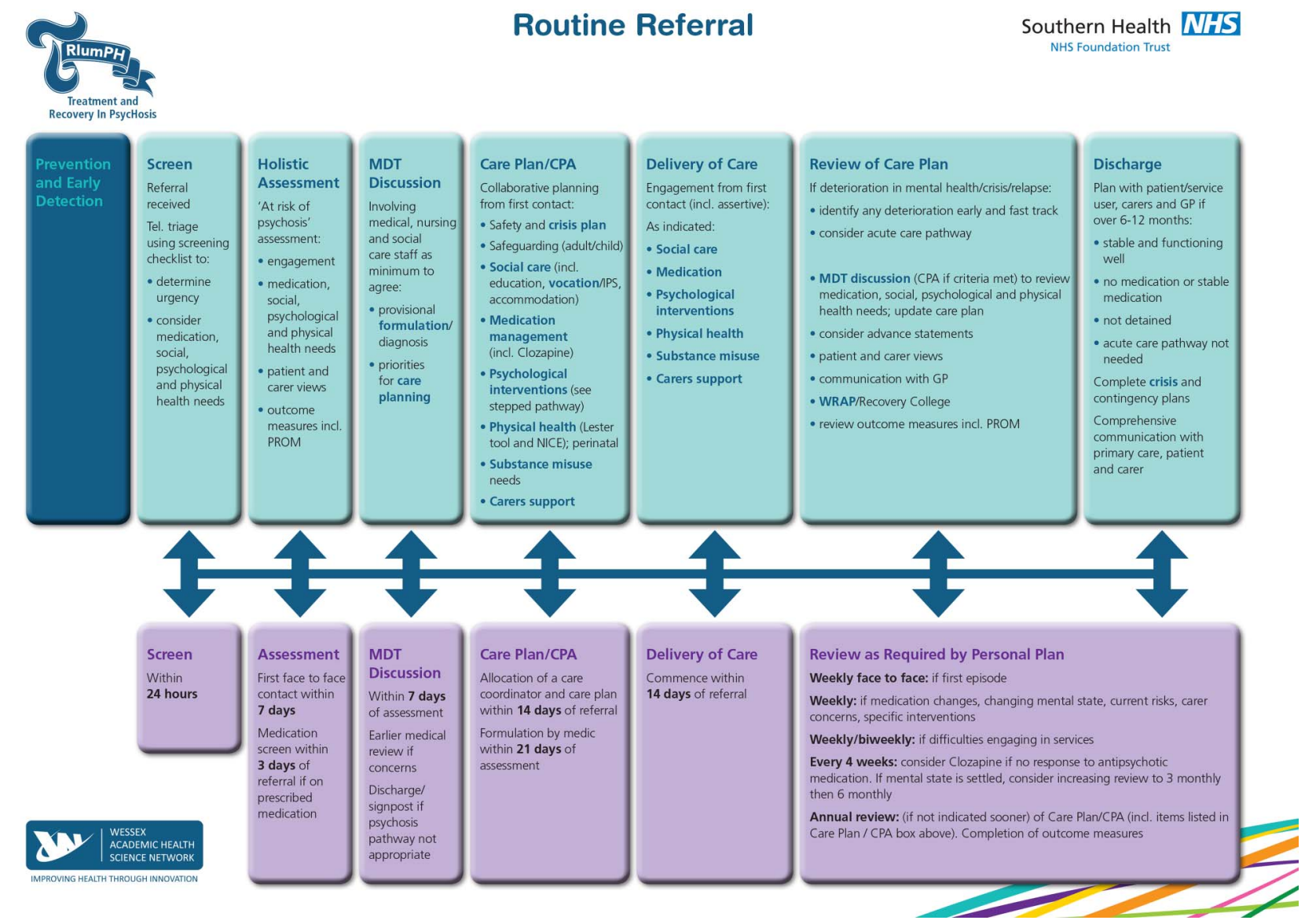

Figure 1 TRlumPH pathway—routine referral. 


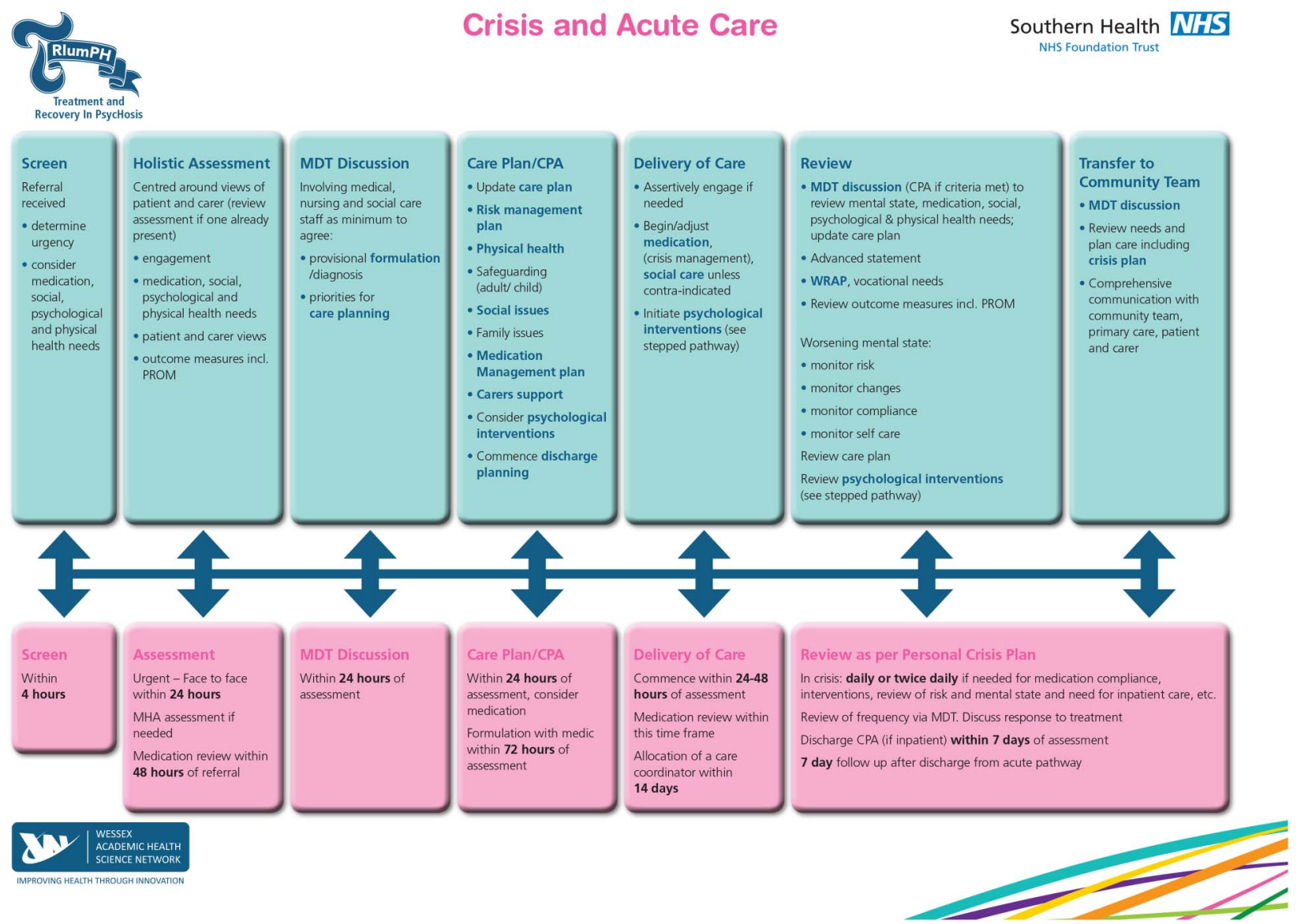

Figure 2 TRlumPH pathway—crisis pathway.

opportunities and challenges of current service provision and understand how 'experts by lived experience' would like to see services improve. Southampton voluntary services (UK) provided rooms for the workshops that were attended by integrated stakeholders-20 patients from across Hampshire, 3 carers and 5 healthcare professionals-and lasted 4 hours. The workshops were facilitated by Stripes partners (a global strategy and innovation studio that support organisations to understand their customers better by providing a fresh perspective and a deep understanding ie, then shared by the team collectively) to establish a 'bottom-up' understanding of priorities specific to Wessex.

Following an ice breaker exercise, participants were asked to write about their 'hopes and fears.' In the second stage, rather than start with any assumptions, the facilitators let participants define what was important to them by asking them to write postcards about a chosen experience and address it to a person who they felt should hear their story. Hundreds of postcards were obtained, yielding many first-hand experiences of current care. The feedback was then collated into themes by the facilitators. The themes were then rated by individual participants in terms of importance and experiences in different settings. ${ }^{23}$ Below are the key themes rated as high priority with examples of quotes from the participants:
- Prevention: 'I was introduced to the system after I committed a crime. It could have been avoided if I had received the right support and intervention'.

- Consistent: 'My brother always complains that his carers and Psychiatrists change all the time, he tells one person all about himself and then, some months or weeks later, he has to tell it all over again. He says he gets fed up with this'.

- Responsive: 'If services intervened early, hospitalisation could have been avoided. If I had been able to get rapid response might also have avoided crisis'.

- Empathetic: 'My husband called the hospital at home team and he did not feel listened to'.

- Seamless: 'The most difficult aspect of my care is that since I moved there is little communication between agencies'.

This approach helped us to understand what was intrinsically important to patients and carers, rather than relying on assumptions.

The pathway attempts to address the key areas, as standardising care improves quality through multidisciplinary communication using care planning that makes care consistent, seamless and responsive. The pathway emphasises prevention through communication with primary care and reduction in DUP; it emphasises consistency and seamless care through a prescribed standardised approach in every phase; responsiveness through 
early access and interventions. Through a standardised approach to assessment and treatment, it promotes empathy.

\section{Engagement with clinicians and stakeholders}

Wider engagement events were held with patients, carers, clinicians, managers and wider stakeholders like commissioners, academics and these also gained political support. Feedback can be viewed on: http:// wessexahsn.org.uk/programmes/show/5/mental-health A steering group was then set up, involving clinicians, commissioners, patient and carer representatives, general practitioners and other stakeholders who were essential to the development of the pathway. Information from the acquired data and coproduction workshops were triangulated with clinical expertise and national evidence to chart process maps. ${ }^{24}$ The process map identified the scope of the pathway and key outcomes desired, including the specific timeframes for access and intervention and highlight the potential challenges and opportunities. ${ }^{23}$

The pathway has been implemented in the EIP teams of two organisations, called the adopter sites. In implementing the pathway, a multipronged approach has been used, including training of EIP team clinicians and development of a guide supporting the pathway; establishment of a pathway facilitator and collection and discussion of live data in clinical teams.

\section{EIP team training and guide}

A guide on how to use the pathway has been produced and placed on the trust website. All participant EIP teams in adopter sites have received information and training on different aspects of implementing the pathway. Training has followed the philosophy and psychological principles that underpin quality improvement initiatives. Training has also been provided on key aspects of care like how to conduct physical health examination; how to deal with comorbidity of alcohol and substance misuse among other aspects.

\section{Pathway facilitator}

A pathway facilitator role has been established within the EIP teams. The facilitator supports the EIP teams in adopter sites to implement the pathway and maintain consistency and fidelity to the model.

\section{Continuous improvement through use of data}

Regular data collection and feedback to clinicians, managers and the pathway steering group has been informing effective implementation of the pathway.

This pathway needs to be evaluated for its feasibility, acceptability, effectiveness and direct costs. Therefore, this study will:

1. Assess the feasibility of implementing the pathway from the perspective of patients, carers and staff

2. Measure patient outcomes to assess effectiveness of the pathway
3. Assess the acceptability of the pathway to patients, carers and clinicians.

4. Evaluate direct costs (eg, care cost, hospital bed costs etc) of the pathway

\section{Control-care as usual}

Participants in the control arm will receive care as usual. There is a significant variability in provision of care, despite NICE guidelines on treatment and management of first episode psychosis. ${ }^{14}$ The study team will record any uptake of NICE recommended programmes during the study, and collate information from centres about uptake of NICE recommendations more widely. These organisations are similar to the adopter sites in that they aspire to follow the EIP policy implementation guide and meet the access and waiting time (AWT) standard. There are also differences as there is a variability in the way care is provided in each team and organisation depending on culture of the team, resources allocated and leadership.

\section{Outcome measures}

Feasibility and acceptability of the pathway will be assessed through quantitative and qualitative data collection regarding adherence to the process measures of the pathway, including timeliness of access and intervention; type of intervention offered, including medication, physical health assessment, psychological intervention etc and reasons for deviation from the pathway. Additionally, satisfaction and acceptability will be assessed using questionnaires and focus groups consisting of the following qualitative methods; patient experience using specifically designed patient experience questionnaires and focus groups, staff experience using staff questionnaires designed to measure the impact of the pathway on staff experience and carer experience using carer focus groups.

Effectiveness will be assessed through data collection and evaluation in patient outcomes, including clinical (eg, HoNOS: Health of Nation Outcome Score), functional and recovery outcomes (eg, employment, vocation, physical health), among other measures. Analysis of direct costs will be measured through a change in service use (eg, routinely collected data on crisis, admissions, detentions, Emergency Department (ED) attendances). See table 1 for full details.

All outcome measures will be assessed at baseline and after 12 and 24 months to measure if there is an effect and if so, whether this is sustained over the longer term. Outcomes measures at the adopter sites will be compared to their own baseline and against comparator sites.

\section{Sample size}

As this is a feasibility prospective study, no a priori power and sample size calculations have been performed. Instead, data for all available patients and staff during 
Table 1 TRlumPH study quantitative outcome measures

\begin{tabular}{ll}
\hline Demographic data (age, gender, ethnicity, employment, housing) & Acute care \\
Process outcomes & Contact acute MH services (in EIP) \\
Referral source & Date acute mental health admission \\
Central triage point & Acute mental health service/Inpatient Screening \\
& within 4hrs \\
Time from referral to assessment & MHA (Mental Health Act) information \\
DNA's (did not attend) prior to assessment & Date of discharge from acute care \\
Time to allocation and engagement by care coordinator & Date crisis plan completed \\
Time to multidisciplinary team (MDT) discussion & Number of subsequent acute admissions \\
Time to medical formulation & Date of attendance at ED \\
Time to CPA (care plan approach)/care plan & Reason for ED attendance \\
Time from referral to intervention offered & Date of general hospital admission (in EIP) \\
Type of intervention (NICE approved) & Date general hospital discharge \\
Time to carer support assessment offered & Contact with criminal justice system \\
Time to ISP (individual placement and support)/vocational support & Date of conviction \\
assessment & Clinical, functional and recovery outcomes \\
Time to risk assessment completion & HoNOS (Health of Nation Outcome Score) \\
Time to discharge from EIP & Change in employment/vocational status \\
Reason for discharge from EIP & Reduction in number of admissions, length of stays \\
Physical health & Reduction in ED attendance \\
General physical health information & \\
Smoking status and intervention & \\
Alcohol status and intervention & \\
Weight assessment and management & \\
Waist circumference & \\
Fasting glucose & \\
Lipid profile & \\
Substance use assessment and management & \\
Interventions & \\
Offered cognitive-behaviour therapy & \\
Offered family intervention & \\
Offered employment support & \\
\hline
\end{tabular}

the study period as a convenience sample of consecutive participants will be used.

\section{Selection criteria}

1. Routinely collected data on every patient that are accepted by the EIP teams in participant organisations during the study period.

2. Patient questionnaires and focus groups:

Participants will be eligible for inclusion in the study if they are:

- On the EIP caseloads,

- Are able to give written informed consent,

- Are able and willing to attend and participate in a focus group,

- Are able to speak and read English.

Participants are excluded from the study if they have a:

- Acute episode of mental illness that could seriously reduce their ability to consent and understand the information,

- Significant alcohol or substance misuse which, in the opinion of the principal investigator, would limit the patient's ability to participate in the study due to lack of ability to understand the information,
- Diagnosis of learning disability that impacts on capacity to consent and understand the information,

3. Staff questionnaires: anonymous questionnaires will be offered to all staff in Adult mental health services in all the participant sites at baseline and at follow-up.

4. Carer focus groups: carers of patients on EIP caseloads who are willing and have the capacity to consent will be invited.

\section{Recruitment of participants for questionnaires}

The study will be promoted within clinical teams and in areas where mental health services are delivered. Research facilitators will work with EIP clinical teams within the participant mental health trusts to identify potentially eligible participants from their caseload for questionnaire surveys and focus groups.

Every patient with psychosis that is accepted by the EIP teams during the study period in the participant teams identified through their care workers and those who meet the inclusion criteria will receive the questionnaire. During the study, no personal information will be collected other than a person's name for consent purposes and telephone number for contact purposes. Every staff member from adult mental health in the 
participant teams will be invited to participate in the anonymised survey.

\section{Recruitment of participants to focus groups}

Patient, carer and staff focus groups will be conducted. A purposive sample will be recruited of individuals who meet the inclusion criteria. On the basis of previous qualitative research involving individuals with schizophrenia, ${ }^{25}$ it is expected that $15-20$ individuals for each set of stakeholders will need to be recruited for this study to reach a point of data saturation (ie, no new insights can be gained and information provided begins to repeat itself). Focus groups will be composed of $6-8$ individuals. $^{26}$

\section{Patients}

Initial contact with potential participants will be made by care coordinators or care professionals. Interested individuals will then be contacted by the research team via phone or in person. In other cases, interested individuals may contact the research team directly, through self-referral via a study advertisement, by phone or in person. Study advertisements will be posted in the participant sites. After contact has been established, individuals will meet with the research team to discuss the study and be provided with an informational sheet. If the individual is interested in participating in the study, informed consent will then be obtained. Individuals will be given as much time as needed to determine whether they would like to participate in the study.

\section{Family/caregivers}

Interested carers of people who are part of the pathway will be contacted via care coordinators or they may contact the research team directly, through self-referral via a study advertisement, by phone or in person. Study advertisements will be posted in the participant sites. After contact has been established, individuals will meet with the research team to discuss the study and be provided with an informational sheet. If the individual is interested in participating in the study, informed consent will then be obtained. Individuals will be given as much time as needed to determine whether they would like to participate in the study. Informed consent will be obtained prior to the focus group taking place.

\section{Clinicians}

Interested individuals may contact the research team directly, through self-referral via a study advertisement, by phone or in person. Study advertisements will be posted in the participant sites. After contact has been established, individuals will meet with the research team to discuss the study and be provided with an informational sheet. If the individual is interested in participating in the study, informed consent will then be obtained.

\section{Focus group procedures}

Participants will be invited to participate in a focus group in a quiet room. A safe environment will be chosen which is known to the individuals. Focus groups will be led by staff trained in conducting qualitative studies involving people with psychosis. Written informed consent will be sought from participants. Once consent has been received, the participants will participate in a 1-2 hour $(\max )$ focus group. At the beginning of the focus group, participants will be asked to fill out a demographic information sheet.

Participants may withdraw themselves from the focus groups, if they so choose. If a participant chooses to withdraw after a focus group starts, it will not be possible to remove their data.

\section{Analysis of data}

The distribution of the quantitative variables will be tested by Kolmogorov-Smirnov or Shapiro-Wilk tests, as appropriate. If their distribution differs from the Gaussian (normal) distribution, they will be presented by median and IQR. Continuous variables (eg, the questionnaire scores at baseline) will be compared among centres using t-tests while categorical variables (eg, gender) will be compared using $\chi^{2}$ or Fisher's test. End of therapy scores on various outcome measures before and after the intervention will be measured using paired t-test. A process evaluation will be undertaken 'to explain discrepancies between expected and observed outcomes, to understand how context influences outcomes, and to provide insights to aid implementation'. ${ }^{27}$ Differences between the Intervention and Control groups will be measured using a linear regression. The statistical software package SPSS will be used for all evaluations as licenced to the Southern Health NHS Foundation Trust.

Comparison between pre-intervention and post intervention scores in satisfaction questionnaire will be made by parametric and non-parametric tests. Where required question analysis will be used to analyse data for emerging themes.

\section{Qualitative data analysis}

Qualitative analysis will be used to explore focus group discussions on the acceptability of the psychosis pathway to patients, carers and staff as well as their range of behaviours and perceptions, including barriers to its implementation. Thematic content analysis will be used to identify emerging themes from focus group and individual interviews with staff, carers and patients. Data will be audio-recorded and transcribed verbatim and emergent themes generated. Data analysis will involve the researchers immersing themselves in data by reading and re-reading the interview transcripts to identify emerging themes. Field notes will also be analysed for nonverbal communication and behaviours and compared to interview data. Each group will consist of six to eight participants who will be approached by researchers, 
experienced in qualitative research. $\mathrm{NViVO}-\mathrm{a}$ qualitative data management software package-will be used to store and code data. Content data will be recorded and transferred for analysis using NVivo. Triangulation of themes will be conducted to assess reliability and validity of the analysis. Independent researchers will review a selection of transcripts to check for accuracy in coding of themes and reliability of interpretation. Verbatim extracts will be included to support interpretation of emergent themes.

\section{Evaluation of direct costs}

The evaluation of direct costs will be from a health and social care and societal perspective. It will consist of cost analysis for estimation of direct costs of care (eg, average costs such as inpatient stay costs, crisis costs, ED costs etc).

\section{Data protection}

The raw data (digital recordings, transcripts) will be stored in encrypted form on a password-protected network at the research department in Southern Health NHS Foundation Trust. Consent documents, demographic forms, receipts for reimbursement of travel will be stored in a locked filing cabinet in the department. Only research staff will have access to the above facilities.

Original consent forms from service users and staff who wishes to take part in the interviews will be sent to the research department in Southern Health NHS Foundation Trust, and kept in the Study Master File.

Data will only be handled by senior trust staff who are DBS checked and will comply with the Data protection act and Information Governance policy of the trust. All data will be completely anonymised for subsequent graphical and statistical analyses.

\section{STUDY SUPERVISION}

Southern Health NHS Foundation Trust is the sponsor for the study and, therefore, has overall responsibility for the study along with the Chief Investigator (CI). Following groups govern the conduct of this study:

- Psychosis pathway steering group: This group is chaired by the first author who is also CI of the study. The membership includes key stakeholders, including clinicians, managers, patient representative, commissioners, Healthwatch representative, pathway facilitator, a primary care representative and a representative from the AHSN. Key strategies for successful implementation and monitoring are planned in this group.

- Data Monitoring and Ethics group: This group is attended by the CI, pathway facilitator, a representative from AHSN, research department manager, statistician and study manager. Data and ethics of the study are discussed in this group.

- Study management group: This group is attended by the study team, including CI, study manager, research facilitators, pathway facilitator, representatives from the participant sites, including principal investigators and trial facilitators. The Study Management Group oversees the day-to-day management of the study.

\section{ETHICAL CONSIDERATIONS}

The ethical issues in this study will be related to the identification and recruitment of patients, the procedure for gaining fully informed consent and data protection arrangements. Written informed consent will be obtained from every participant.

Ethics approval for this study was obtained from East of Scotland Research Ethics Service (EoSRES) (REC Ref no. LR/15/ES/0091).

\section{Declaration of Helsinki}

This study complies, and at all times will comply, with The Declaration of Helsinki, as adopted at the 18th World Medical Association (WMA) General Assembly, Helsinki, Finland, 1964 and last amended at the 64th WMA General Assembly, Fortaleza, Brazil, October 2013.

\section{DISSEMINATION OF STUDY RESULTS}

The results will be disseminated through publications, conference presentations, and reports to the organisation.

\section{Safety assessments}

The development of the pathway ensured that it was tailored to the needs of people with psychosis. As the intervention is a service improvement, no adverse events are anticipated as a direct result of implementation of the pathway.

\section{DISCUSSION}

Evidenced-based integrated pathways have been used successfully in stroke and cardiovascular illness. They provide a standardised framework for good clinical practice, reduce variation in care and have improved outcomes for patients through providing timely access and intervention. Standardised pathways improve quality by improving multidisciplinary communication with different care agencies using care planning, and improve patient satisfaction. ${ }^{22}$ While currently there are pathways and guidance available for psychosis care in some countries such as UK, ${ }^{14}$ they do not provide prescriptive timeframes to improve access to care and interventions. In the UK, the access and waiting time standard ${ }^{28}$ has been introduced to begin to address these issues and reduce the DUP in first episode psychosis. However, an Integrated Care Pathway provides far more benefits than meeting a target in terms of providing personalised good quality care.

\section{Development of the pathway}

The strength of the Integrated Care Pathway TRIumPH is in its development through inspiring shared vision 
and purpose, patient empowerment through coproduction and codesign; intelligent use of information; influencing; and systems networking across interfaces through stakeholder engagement, to incorporate diverse views and impact on quality outcomes for patient care. Codesigning services with clinicians and patients together makes a difference to quality improvement, as it draws from the expertise of patients, who are experts by experience and professional leaders. ${ }^{29}$ The recent Mental Health Taskforce report ${ }^{30}$ recommends that Integrated Care Pathways are developed for mental health conditions to reduce fragmented care and improve outcomes.

\section{Evaluation}

There is a huge potential to improve quality and productivity in mental health services. ${ }^{31}{ }^{32}$ Across the world initiatives have been taken in community services but there are few evaluations of effectiveness or efficiency. ${ }^{33}$ There is also a need for standardisation and guidelines on conducting such studies.

If the results of the study show that the pathway is feasible, of benefit to patients, carers and staff, the study protocol will set a benchmark against which organisations can organise and audit their practice, which can also be used to evaluate the effectiveness of service changes. For practitioners, they will provide practical guidance about what to do and when to do it, making best use of their professional skills. The pathway can also assist in establishing workforce and training need, such as professional, therapy, vocational and support worker time and training.

\section{Limitations}

Most organisations are currently struggling to balance the quality versus finance agenda due to ongoing austerity. Limited resources within services to deliver care along the pathway may impact on results. These may include inadequate numbers of clinicians in teams with an understanding of the pathway and trained therapists to deliver the interventions. Adaptation and adoption of the pathway in other developed countries like the United States and Canada may have challenges due to system differences, hospital-based care and different payment systems. Similarly, there maybe challenges in developing countries where care is hospital-based and attributions to illness and help-seeking pathways into care are culturally influenced.

\section{CONCLUSION}

In conclusion, the TRIumPH study will provide evidence for the feasibility, acceptability and impact of an Integrated Care Pathway that has been developed through intelligent use of information, coproduction with patients, carers and clinicians and engagement with wider stakeholders. If the pathway is successful, the design of the intervention and study will allow a rapid dissemination within the NHS.

\section{Author affiliations}

${ }^{1}$ Southern Health NHS Foundation Trust, Calmore, Southampton, UK ${ }^{2}$ Wessex Academic Health Sciences Network, Chilworth, Hampshire, UK

${ }^{3}$ University of Southampton, Southampton, UK

${ }^{4}$ Solent NHS Trust, Southampton, UK

${ }^{5}$ Queens University, Kingston, Canada

Contributors SR and DK are grant holders and contributed to the concept and design of the study. SR is the $\mathrm{Cl}$ and led the development of the study protocol and study documentation, and made a significant contribution to the manuscript. All authors contributed to elaboration and refinement of the study protocol and approved the final manuscript.

Funding This project has been funded by the NHS England (Regional Innovation Fund programme); Small grant from the Royal College of Psychiatrists general adult faculty. The production of the pathway has been supported by the Wessex Academic Health Sciences Network (AHSN) and evaluation has also been supported by the Wessex Clinical Research Network (CRN). The views and opinions expressed therein are those of the authors and do not necessarily reflect those of funders, NIHR, NHS or the RCPsych, AHSN or CRN. The study is sponsored by Southern Health NHS Foundation Trust.

Competing interests None declared.

Provenance and peer review Not commissioned; externally peer reviewed.

Study status The study is currently ongoing.

Open Access This is an Open Access article distributed in accordance with the Creative Commons Attribution Non Commercial (CC BY-NC 4.0) license, which permits others to distribute, remix, adapt, build upon this work noncommercially, and license their derivative works on different terms, provided the original work is properly cited and the use is non-commercial. See: http:// creativecommons.org/licenses/by-nc/4.0/

\section{REFERENCES}

1. Kirkbride JB, Errazuriz A, Croudace, et al. Incidence of schizophrenia and other psychoses in England, 1950-2009: a systematic review and meta-analyses. PLOS ONE 2012;7: e31660.

2. Baldwin P, Brownie D, Scully $P$, et al. Epidemiology of first-episode psychosis: illustrating the challenges across diagnostic boundaries through the Cavan-Monaghan study at 8 years. Schizophr Bull 2005;31:624-38.

3. Brown S, Kim M, Mitchell C, et al. Twenty-five year mortality of a community cohort with schizophrenia. Br J Psychiatry 2010;196:116-21.

4. World Health Organization. The World Health Report 2001. Mental Health: New Understanding, New Hope. WHO, 2001.

5. Rössler W, Salize HJ, Van Os J, et al. Size of burden of schizophrenia and psychotic disorders. Eur Neuropsychopharmacol 2005;15:399-409.

6. McGorry PD, Edwards J, Mihalopoulos C, et al. EPPIC: an evolving system of early detection and optimal management. Schizophr Bull 1996;22:305-26.

7. Birchwood Todd P, Jackson C. Early intervention in psychosis. The critical period hypothesis. Br J Psychiatry 1998;172(Suppl 33):53-9.

8. Drake R, Haley C, Akhtar S, et al. Causes and consequences of du-ration of untreated psychosis in schizophrenia. Br J Psychiatry 2000;177:511-15.

9. McGlashan TH. Duration of untreated psychosis in first-episode schizophrenia: marker or determinant of course? Biol Psychiatry 1999;46:899-907.

10. Marshall M, Lewis S, Lockwood A, et al. Association between duration of untreated psychosis and outcome in cohorts of first-episode patients. A systematic review. Arch Gen Psychiatry 2005;62:975-83.

11. Connor C, Greenfield S, Lester H, et al. Seeking help for first-episode psychosis: a family narrative. Early Interv Psychiatry 2014;10:334-45.

12. Rathod S, Phiri P, Kingdon D, et al. Developing culturally sensitive cognitive behaviour therapy for psychosis for ethnic minority patients 
by exploration and incorporation of service users' and health professionals' views and opinions. Behav Cogn Psychother 2010;38:511-33.

13. Rathod S, Kingdon D, Pinninti N, et al. Cultural adaptation of CBT for serious mental illness: a guide for training and practice. WileyBlackwell, 2015.

14. National Institute for Health and Care Excellence. Psychosis and schizophrenia: treatment and management. 2014 (Clinical guideline 178). http://guidance.nice.org.uk/CG178.

15. The Schizophrenia Commission (2012). The abandoned illness: a report from the Schizophrenia Commission. London: Rethink Mental Illness. Ref 30: NHS England, The Mental Health Taskforce, March 2015.

16. Royal College of Psychiatrists. Report of the National Audit of Schizophrenia (NAS) 2012. London: Healthcare Quality Improvement Partnership.

17. Rethink Mental Illness. Lost generation: protecting early intervention in psy-chosis services. Rethink Mental Illness. London, 2014

18. Lloyd-Evans B, Crosby M, Stockton S, et al. Initiatives to shorten duration of untreated psychosis: systematic review. Br J Psychiatry 2011;198:256-63.

19. Lester H, Birchwood M, Bryan S, et al. Development and implementation of early intervention services for young people with psychosis: case study. Br J Psychiatry. 2009;194:446-50.

20. Birchwood M, Connor $\mathrm{C}$, Lester $\mathrm{H}$, et al. Reducing DUP in first-episode psychosis: care pathways to early intervention in psychosis teams. Br J Psychiatry 2013;202:1-7.

21. Norman RM, Malla AK. Duration of untreated psychosis: a critical examination of the concept and its importance. Psychol Med 2001;31:381-400.

22. Campbell $\mathrm{H}$, Hotchkiss $\mathrm{R}$, Bradshaw $\mathrm{N}$, et al. Integrated care pathways. BMJ 1998;316:133.

23. Rathod S, Griffiths A, Kingdon D, et al. Pathways to recovery: a case for adoption of systematic pathways in psychosis. Jointly produced by Imperial College Health Partners and Wessex Academic Health Science Network; Supported by the Royal College of Psychiatrists and Rethink Mental Illness. 2015

24. Middleton S, Roberts A. Clinical pathways workbook. Wrexham: VFM Unit, 1998:6.

25. Faulkner G, Gorczynski P, Cohn T. Psychiatric illness and obesity: recognizing the "Obesogenic" nature of an inpatient psychiatric setting. Psychiatr Serv 2009;60:538-41.

26. Krueger RA. Focus groups: a practical guide for applied research. 2nd edn. Thousand Oaks, CA: Sage, 1994.

27. Craig P, Dieppe $\mathrm{P}$, Macintyre S, et al. Developing and evaluating complex interventions: the new Medical Research Council guidance. BMJ 2008;337:a1655

28. NHS England. Guidance to support the introduction of access and waiting time standards for mental health services. 2015.

29. Bate $P$, Robert $G$. Experience-based design: from redesigning the system around the patient to co-designing services with the patient. Qual Saf Health Care 2006;15:307-10.

30. NHS England. Mental Health Taskforce Strategy-The five year forward view for Mental Health. 2016.

31. Royal College of Psychiatrists. Looking Ahead. Future development of UK mental health services: Recommendations from a Royal College of Psychiatrists' enquiry. Occasional Paper OP75. London, 2010a.

32. WHO. Expert opinion on barriers and facilitating factors for the implementation of existing mental health knowledge in mental health services. Geneva: World Health Organization, 2007.

33. Pedersen PB, Kolstad A. De-institutionalisation and trans-institutionalisation-changing trends of inpatient care in Norwegian mental health institutions 1950-2007. Int J Ment Health Syst 2009;3:28. 